\title{
The association of maternal diabetes with attention deficit and hyperactivity disorder in offspring: a meta-analysis
}

This article was published in the following Dove Medical Press journal: Neuropsychiatric Disease and Treatment

\author{
Lifeng Zhao' \\ Xuesong $\mathrm{Li}^{2}$ \\ Guanying Liu' \\ Baoling Han' \\ Jian Wang' \\ Xia Jiang' \\ 'Department of Endocrinology, \\ Tianjin First Center Hospital, Tianjin, \\ China; ${ }^{2}$ First Center Clinical Medical \\ Institute, Tianjin Medical University, \\ Tianjin, China
}

Objective: Recent controversial evidence suggests that maternal diabetes may increase the risk of attention deficit and hyperactivity disorder (ADHD) in offspring. To examine this potential association, a systematic literature search and meta-analysis was performed.

Methods: OR or risk ratio (RR) from each study was obtained and combined for evaluating the risk. Six cohort studies and three case-control studies were included in the present study.

Results: The meta-analysis of the highly heterogeneous case-control studies did not find significant association between maternal diabetes and ADHD risk (OR: 1.20, 95\% CI: 0.96-1.49). The combining of the cohort studies demonstrated that offspring of diabetic mothers were at higher risk of ADHD (RR: 1.40, 95\% CI: 1.27-1.54); however, publication bias was identified. When exposure was specified as gestational diabetes mellitus (GDM), GDM exposure increased the risk of ADHD for children by $164 \%(95 \%$ CI: $1.25-5.56)$ in a Caucasian population. Neither heterogeneity nor publication bias was detected.

Conclusion: Maternal diabetes, especially GDM, is probably a risk factor for ADHD in the Caucasian population. More studies based on large sample size and different ethnicities are needed to confirm this association.

Keywords: maternal diabetes, attention deficit hyperactivity disorder, meta-analysis

\section{Introduction}

Attention deficit and hyperactivity disorder (ADHD) is an early-onset neurodevelopmental disorder combining overactivity and impulsivity with the inability to concentrate, resulting in functional impairment in academic, family, and social settings. A systematic review including 175 studies has demonstrated that ADHD affects up to $7.2 \%$ (95\% CI: $6.7-7.8)$ of children worldwide. ${ }^{1}$ A recent investigation in the UK has demonstrated a marked increase in ADHD prevalence, incidence, and medication. ${ }^{2}$ Therefore, this disorder is of particular public health concern. The etiology of ADHD is complex since it is clear that ADHD has a strong genetic component, ${ }^{3}$ while environmental risk factors are also implicated. ${ }^{4}$ For psychiatric disorders in children, exposure to risk factors in utero plays an important role. ${ }^{5}$ For example, a hyperglycemic intrauterine environment may exert a negative impact on the development of fetal brain. ${ }^{6}$

Diabetes before pregnancy is known as pregestational diabetes mellitus (PGDM). Gestational diabetes mellitus (GDM) is defined as diabetes diagnosed in the second and third trimesters of pregnancy. Experiments in animal models of diabetic pregnancies have demonstrated that maternal hyperglycemia usually creates an inflammatory environment by promoting oxidative stress via production of reactive oxygen species
Correspondence: Lifeng Zhao

Department of Endocrinology, Tianjin First Center Hospital, No 24, Fukang Road, Nankai District, Tianjin 300I92, China

Tel +86 I55 22779818

Email hengliyadang.com@163.com 
within the embryo and fetus. ${ }^{7}$ Intriguingly, it has been hypothesized that ADHD may be due to central nervous system inflammatory response in the fetus caused by maternal inflammation and immune response. ${ }^{8}$ In addition, murine models have showed that maternal diabetes induces imbalance in the epigenetic mechanisms, alters transcriptional factors and signaling pathways, and thereby contributes to neurodevelopmental disorders. ${ }^{9}$

In line with the experimental evidence mentioned earlier, population studies have suggested a possible association between maternal diabetes and the risk of ADHD for children. Several observational studies in the Caucasian and Chinese populations have revealed that maternal diabetes was related to higher risk of ADHD among children. ${ }^{10-13}$ However, some retrospective studies based on large sample size have reported statistically insignificant results, ${ }^{8,14}$ indicating that offspring from diabetic mothers may be vulnerable to ADHD. Because results from population investigations are currently controversial, we systematically searched the electronic databases, identified all the relevant studies, and performed meta-analyses to quantitatively synthesize all the available data. This is, to our knowledge, the first meta-analysis to study the association between maternal diabetes and risk of ADHD in offspring.

\section{Materials and methods}

\section{Literature search}

This meta-analysis followed the recommended PRISMA guidelines. ${ }^{15}$ A comprehensive literature search was conducted on PubMed, Web of Science, Embase, PsycINFO, WanFang, and China National Knowledge Infrastructure (CNKI) database. The most recent search was conducted in November 2018. The detailed search syntax for PubMed was as follows: (((maternal OR pregestational OR gestational) AND (diabetes OR “diabetes mellitus" OR hyperglycemia))) AND ("ADHD” OR "attention deficit" OR "hyperactivity syndrome” OR "attention deficit hyperactivity disorder"). The corresponding Chinese characters were used in CNKI database searching.

After the primary records were retrieved from databases, duplicated studies were removed. Then, the remaining records were checked according to titles and abstracts. Irrelevant studies were excluded during this step. After that, the full texts of the rest of the studies were obtained and reviewed in detail for eligibility according to the inclusion criteria. Finally, the qualified studies were included for further analyses.

\section{Inclusion criteria}

The inclusion criteria were established as follows: 1) original observational studies investigating the association between maternal diabetes and risk of ADHD in offspring, and 2) effect size (usually OR or risk ratio [RR]) and its 95\% CI were reported, or the distribution of subjects in each comparison group was given so that the effect size was able to be calculated. Only English and Chinese literature was included. No restriction on diabetes subtype and study design was imposed. Review, editorial, and conference articles were excluded.

\section{Data extraction}

The following items were extracted independently by two reviewers from each study: first author, publication year, study design, characteristics of the participants, diagnostic criteria of ADHD and maternal diabetes, effect size with its 95\% CI (preferentially adjusted effect size), and adjusted confounders. If the effect size was not reported, it was manually calculated from the original data. Any disagreement was resolved by further discussion. Since Caucasian usually includes modern population of Europe and white people in the US, we roughly classified participants from Europe and the US into Caucasian population when performing subgroup analyses.

\section{Statistical analyses}

The heterogeneity across studies was evaluated with $Q$-statistic, and the significance level was defined as $0.1 .^{16}$ Heterogeneity was further measured by $I^{2}$ value and classified into high, medium, or low when $I^{2} \geq 50 \%, 50 \%>$ $I^{2} \geq 25 \%$, or $25 \%>I^{2}$, respectively. ${ }^{17}$ If an $I^{2}$ was smaller than 25\%, Mantel-Hansel's method in fixed-effect model was used to pool outcomes, otherwise data were pooled based on Dersimonian and Laird method in random-effect model. ${ }^{18}$ The effect size and its lower and higher CIs were natural logarithm transformed before data combining, and the result was natural exponential transformed and displayed. The publication bias was evaluated by the Egger's linear regression test statistically. ${ }^{16}$ Sensitivity analysis was performed with omitting each study and observing whether the synthesized result altered significantly. All statistical analyses were conducted by Stata 9.0 (StataCorp LP, College station, TX, USA). All $P$-values were two-sided and identified as significant if $<0.05$, unless otherwise specified.

\section{Results}

\section{Characteristics of the included studies}

As illustrated in Figure 1, a total of nine studies ${ }^{8,10-14,19-21}$ involving 7,218,903 participants were included in the present analysis. The majority of them ${ }^{8,12-14,19,20}$ were published in the recent 2 years. For baseline information, six studies ${ }^{10-13,19,20}$ 


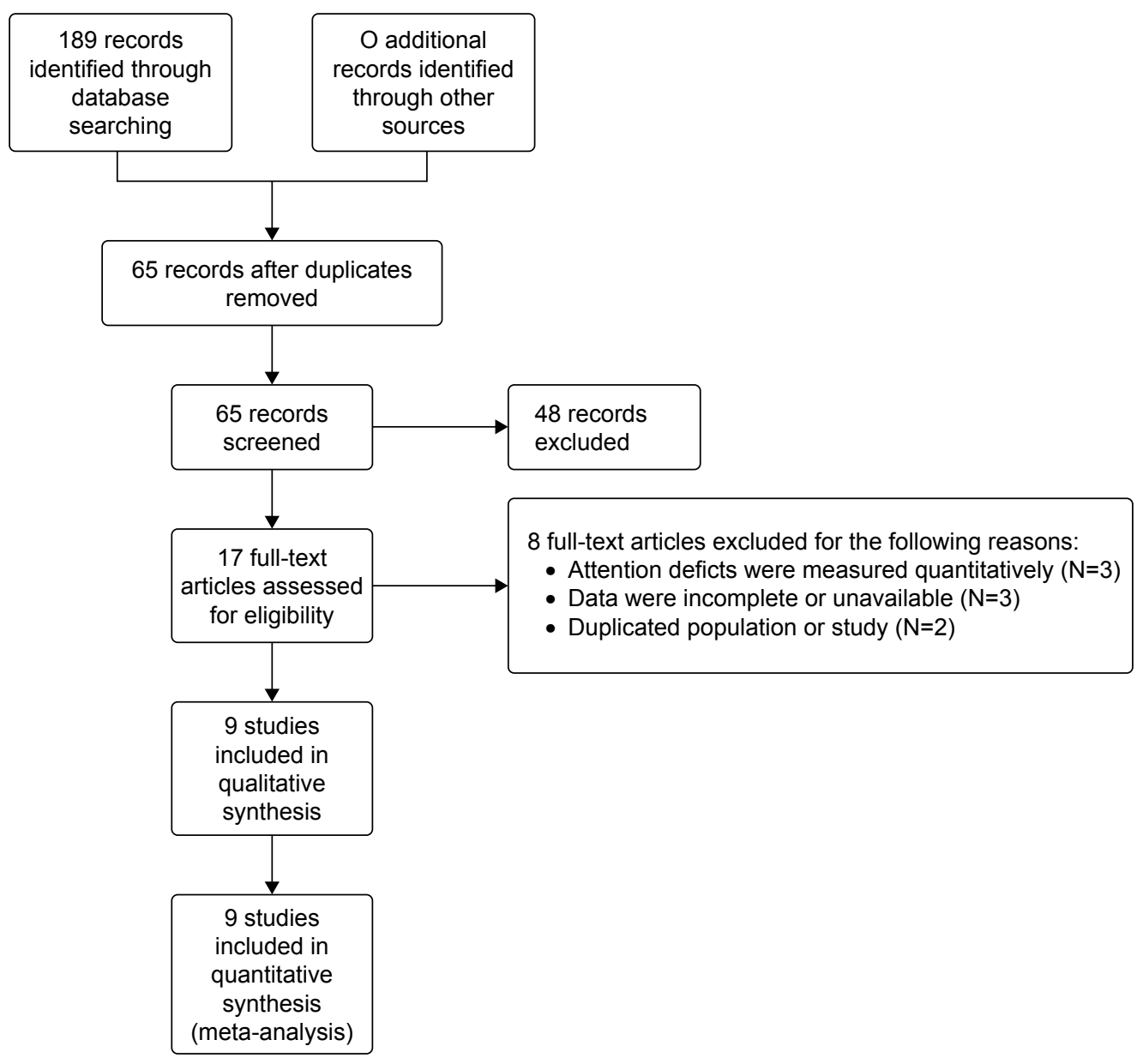

Figure I Flow diagram of the identification of the eligible studies.

were cohort design and used RR or HR to measure the effect size. The remaining three studies ${ }^{8,14,21}$ were case-control studies, using OR to describe the risk. Over half of the studies were conducted in Nordic Europe, ${ }^{8,12-14,19}$ benefiting from the excellent local nationwide registry system. The rest of the studies were from China, ${ }^{11}$ Greece, ${ }^{20}$ Germany, ${ }^{21}$ and USA. ${ }^{10}$ Maternal type 1 diabetes (T1D) was investigated in five studies, ${ }^{8,12-14,19}$ while four studies did not give the accurate type of diabetes..$^{10,11,20,21}$ Only one study recruited mothers with type 2 diabetes (T2D). ${ }^{8}$ Three of the studies ${ }^{10,11,19}$ did not use multivariate analysis in order to consider the potential bias induced by confounders (for details see Table 1). The quality of each study was assessed in Table 2 using NewcastleOttawa Scale for nonrandomized studies. The overall quality levels were evaluated using the GRADE approach in Table 3.

\section{Data synthesis of the case-control studies}

The pooling of data from case-control studies ${ }^{8,14,21}$ demonstrated that maternal diabetes was not associated with ADHD in offspring (OR: 1.20, 95\% CI: 0.96-1.49). Since all the case-control studies were conducted among Caucasian population, the findings should be applied to this race exclusively. High heterogeneity was detected $\left(I^{2}=74.5 \%\right)$ (Figure $2 \mathrm{~A}$ ). Sensitivity analysis indicated that omission of a given study would not reverse the insignificant result (Figure 2B). Egger's test showed that no publication bias existed ( $t=1.67$, $P=0.194)$ (Table 4).

\section{Data synthesis of the cohort studies}

In terms of cohort studies, ${ }^{10-13,19,20}$ the meta-analysis demonstrated that maternal diabetes increased the risk of ADHD in offspring by $40 \%$ (RR: $1.40,95 \%$ CI: $1.27-1.54$ ) (Figure 2C). Sensitivity analysis confirmed that the results were stable (Figure 2D). Unfortunately, publication bias was identified according to Egger's test $(t=6.56, P=0.003)$ (Table 4), indicating that the estimated effect was probably overstated due to publication of positive results. Since only the Li et al's ${ }^{11}$ study investigated the effect of maternal diabetes in Chinese, the remaining studies ${ }^{10,12,13,19,20}$ that were conducted in Caucasian population demonstrated the risk in 


\begin{tabular}{|c|c|c|c|c|c|c|c|c|c|}
\hline 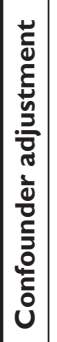 & 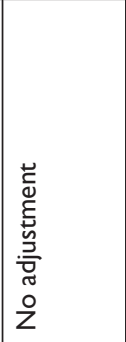 & 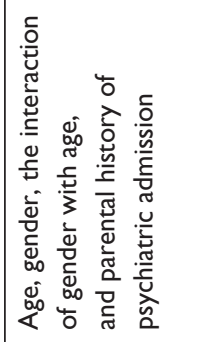 & 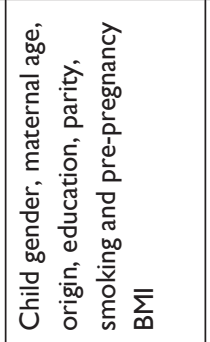 & 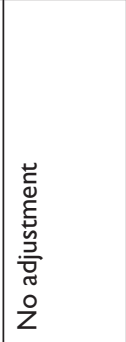 & 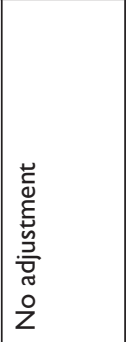 & 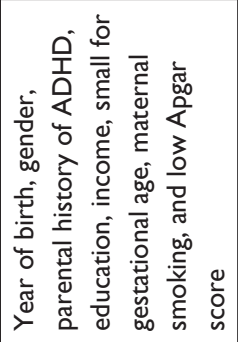 & 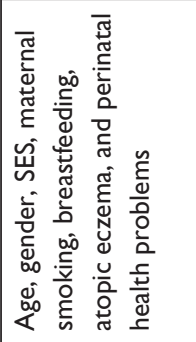 & 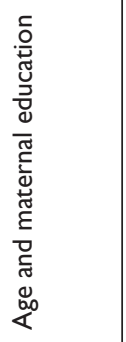 & 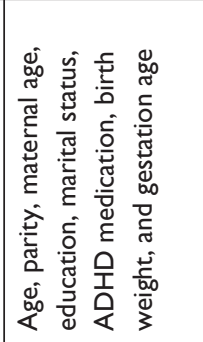 \\
\hline 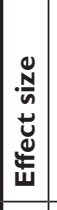 & 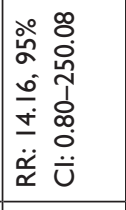 & 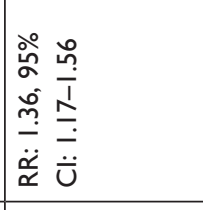 & 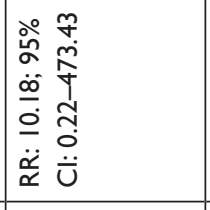 & 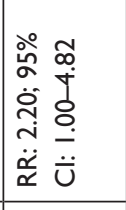 & 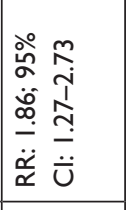 & 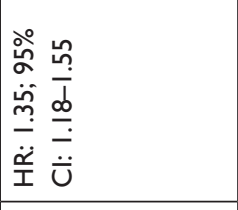 & 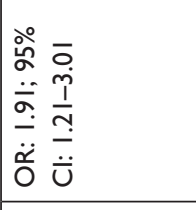 & 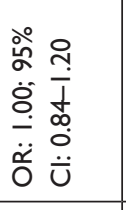 & 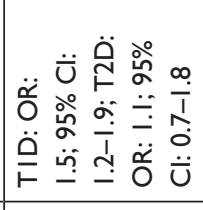 \\
\hline 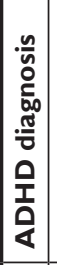 & 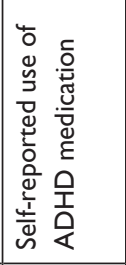 & 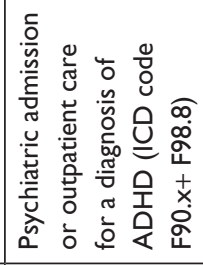 & 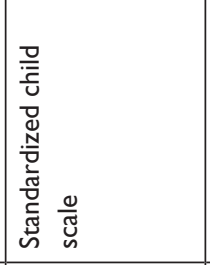 & 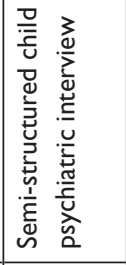 & 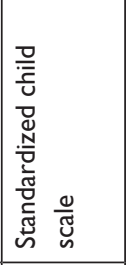 & 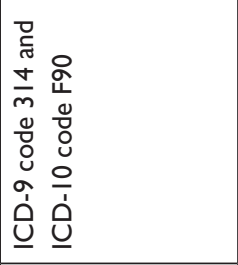 & 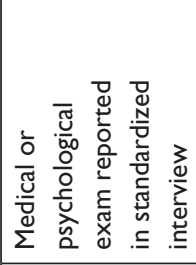 & 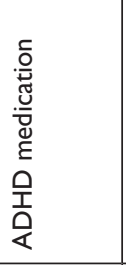 & 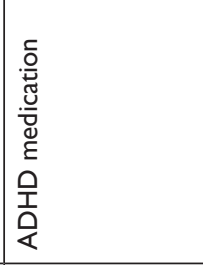 \\
\hline 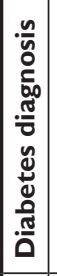 & 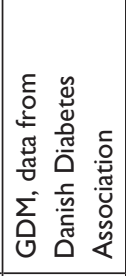 & 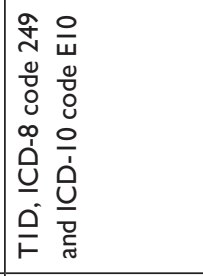 & 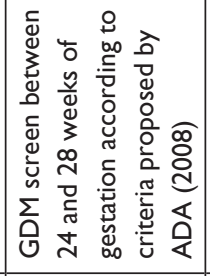 & 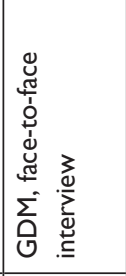 & 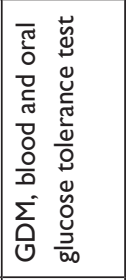 & 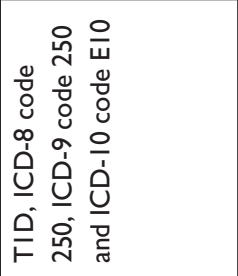 & 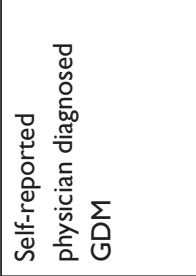 & 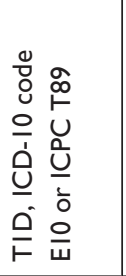 & 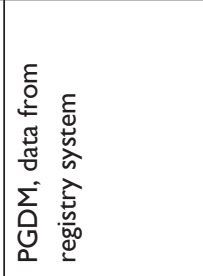 \\
\hline 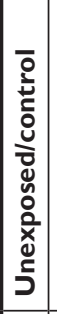 & 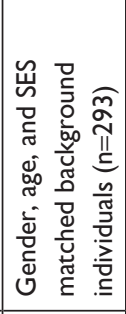 & 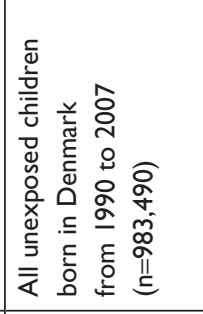 & 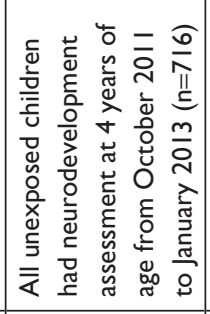 & 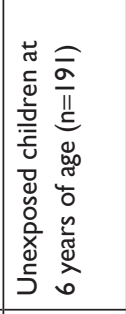 & 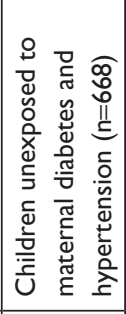 & 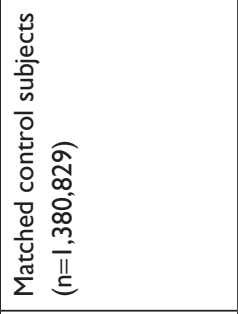 & 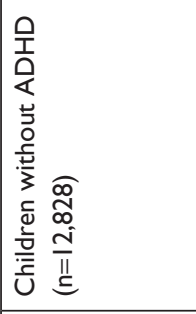 & 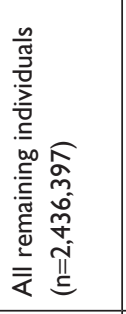 & 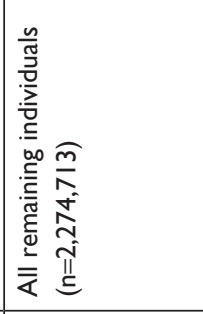 \\
\hline 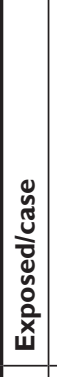 & 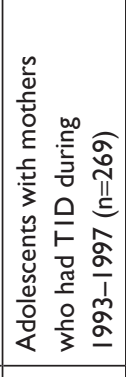 & 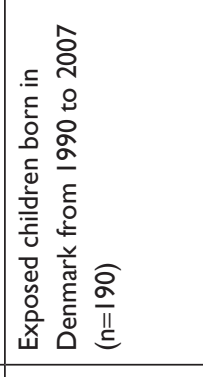 & 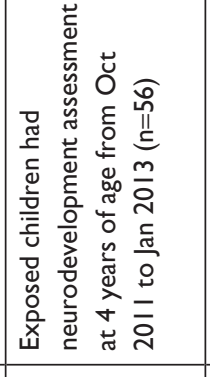 & 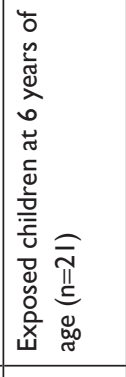 & 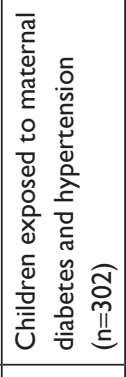 & 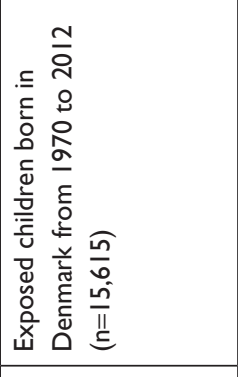 & 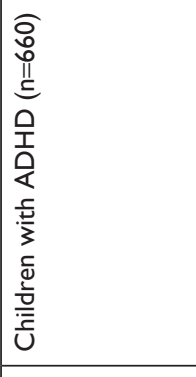 & 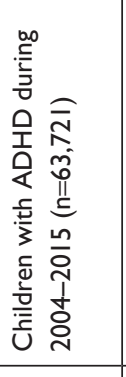 & 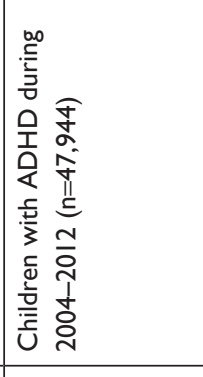 \\
\hline 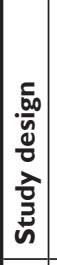 & 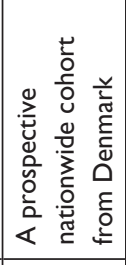 & 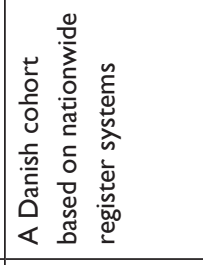 & 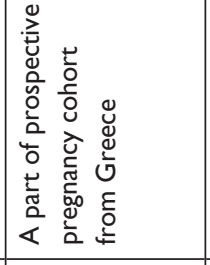 & 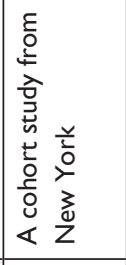 & 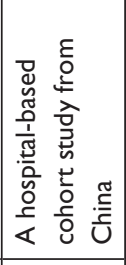 & 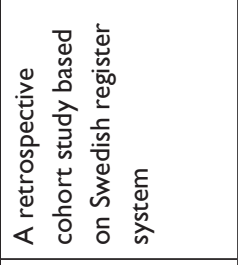 & 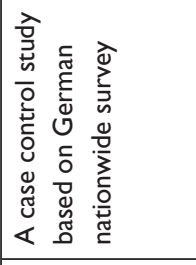 & 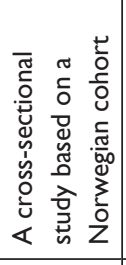 & 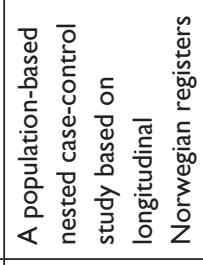 \\
\hline 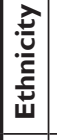 & $u$ & $u$ & $u$ & $u$ & $\varangle$ & $u$ & $u$ & $u$ & $u$ \\
\hline 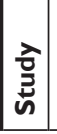 & 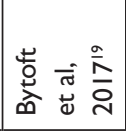 & 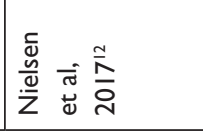 & 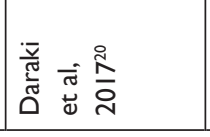 & 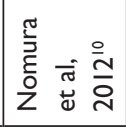 & 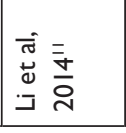 & 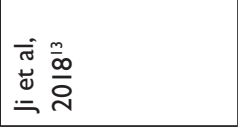 & 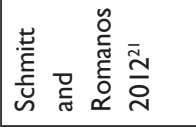 & 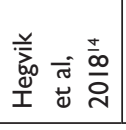 & 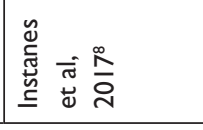 \\
\hline
\end{tabular}


Table 2 Quality of the included studies

\begin{tabular}{|c|c|c|c|c|c|}
\hline Study & Selection score & Comparability score & Outcome score & Total score & Quality \\
\hline Bytoft et al, $2017^{19}$ & 4 & 2 & 3 & 9 & High \\
\hline Nielsen et al, $2017^{12}$ & 3 & 2 & 3 & 8 & High \\
\hline Daraki et al, $2017^{20}$ & 4 & 2 & 3 & 9 & High \\
\hline Nomura et al, $2012^{10}$ & 4 & 2 & 3 & 9 & High \\
\hline Li et al, 2014"I & 3 & 0 & 2 & 5 & Moderate \\
\hline Ji et al, $2018^{13}$ & 3 & 2 & 3 & 8 & High \\
\hline Schmitt and Romanos, 2012 $2^{21}$ & 4 & 2 & 2 & 8 & High \\
\hline Hegvik et al, $2018^{14}$ & 3 & 2 & 3 & 8 & High \\
\hline Instanes et al, $2017^{8}$ & 4 & 2 & 2 & 8 & High \\
\hline
\end{tabular}

this race (RR: 1.37, 95\% CI: 1.24-1.51). Subgroup analyses revealed that T1D increased the risk by $36 \%$ (RR: 1.36, 95\% CI: $1.23-1.50) .{ }^{12,13,19}$ No heterogeneity between studies were found in either overall or subgroup analyses.

\section{Subgroup analysis of the cohort studies investigating mothers with GDM}

Since the participants in the cohort studies consisted of mothers with GDM and mothers with a history of diabetes (the onset of diabetes was not specified), we further narrowed the scope and investigated the effect of GDM on risk of ADHD for children. The combining of four studies ${ }^{10,11,19,20}$ demonstrated that GDM heightened the risk of ADHD by onefold (RR: 2.00; 95\% CI: 1.42-2.81), without detecting inconsistency between studies $\left(I^{2}=0 \%\right)$ (Figure 3A). Publication bias was also identified regarding this analysis. We next excluded Li et al's study ${ }^{11}$ and ensured all the included studies ${ }^{10,19,20}$ were from Caucasian population. The risk of ADHD conferred by GDM in Caucasian was as much as 1.64-fold (95\% CI: 1.25-5.56) higher compared with controls (Figure 3B). Besides, neither heterogeneity nor publication bias was detected (Table 4).

\section{Discussion}

The present study is, to our knowledge, the first metaanalysis to evaluate the risk of ADHD for children induced by maternal diabetes. We systematically searched the databases and some of the included studies were nationwide investigations, and the number of subjects was large enough to obtain sufficient study power. ${ }^{12,13}$ The analyses generally consisted of two parts. On the one hand, combining of case-control studies demonstrated that the offspring of diabetic mothers were not at a heightened risk of ADHD (OR: 1.20, 95\% CI: 0.96-1.49). All the case-control studies recruited Caucasian participants. Therefore, this finding should be restricted to this population. Of note, it has been suggested that some minority groups, such as obese or older Caucasian women, are at a greater risk for maternal diabetes than the overall Caucasian women. ${ }^{22}$ This indicates that management of the high-risk subgroups by maternal DM intervention could be more beneficial for ADHD prevention. On the other hand, cohort studies altogether indicated that maternal diabetes was a risk factor for ADHD (RR: 1.40, 95\% CI: 1.27-1.54). However, this result was not reliable considering publication bias was identified.

Since higher functions of brain develop during the second half of pregnancy, we hypothesized that GDM may exert adverse effects on offspring more profoundly in this period. When the participants were specified as offspring of mothers with GDM in Caucasian population from cohort studies, data pooling suggested that they were more vulnerable to ADHD and the risk was increased as much as by 1.64 -fold (95\% CI: 1.25-5.56). Although the sample size was relatively smaller (number of subjects in exposure group was 515, number of subjects in non-exposure group was 984,499) in this subgroup analysis, neither inconsistency nor publication bias was detected, indicating the reliability of this result. This result emphasizes the importance of good glycemic control in diabetic mothers throughout pregnancy and not only in the first trimester.

It has been hypothesized that ADHD may be due to inflammatory response to the central nervous system in the fetus caused by maternal inflammation and immune response. A nationwide study demonstrated that several immune system diseases, including maternal multiple sclerosis, rheumatoid arthritis, asthma and hypothyroidism, were more frequently observed among mothers of offspring with ADHD compared with mothers of controls. ${ }^{8}$ Apart from the association regardless of gender, another large cross-sectional study reported that inflammatory bowel disease was associated 


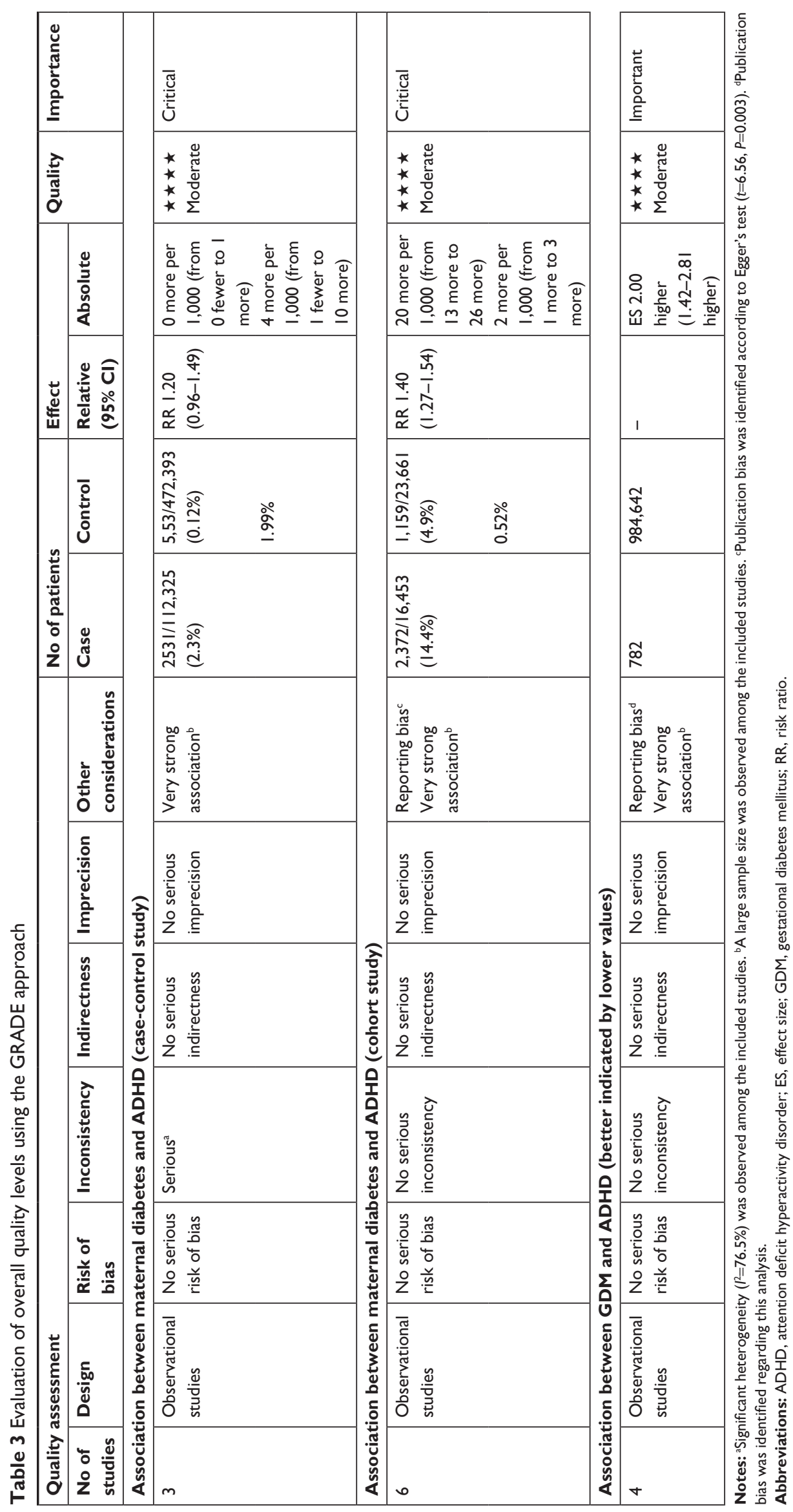


A

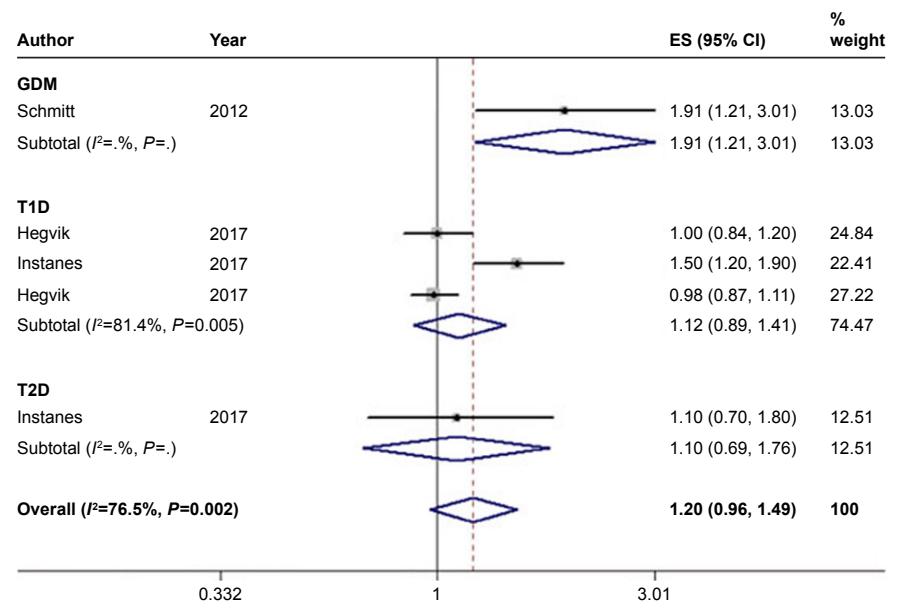

C

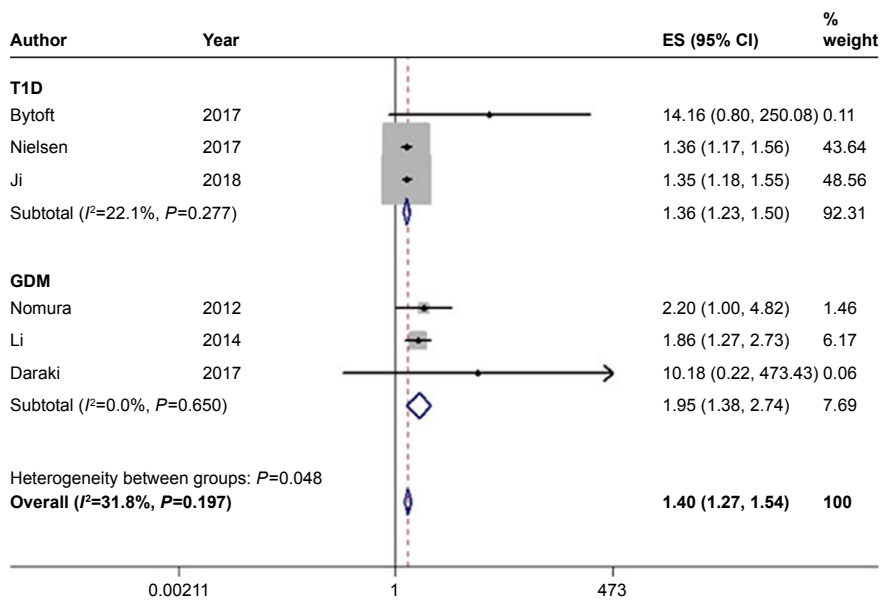

B

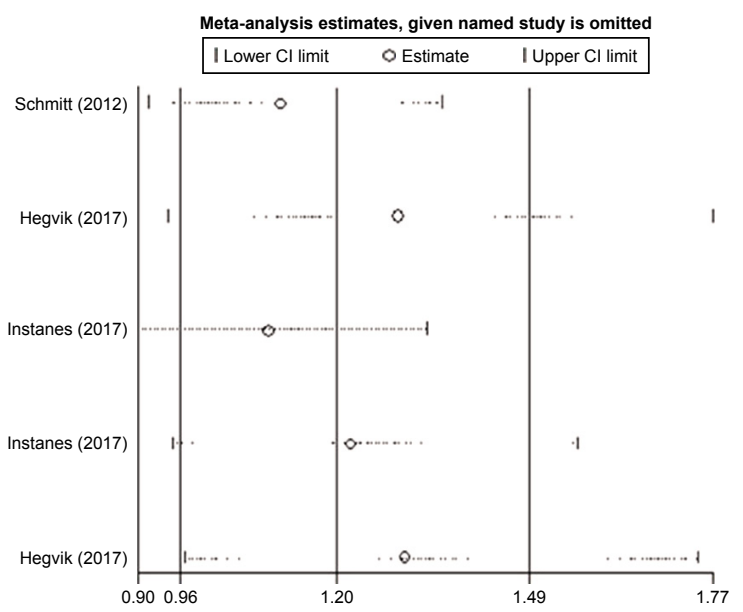

D

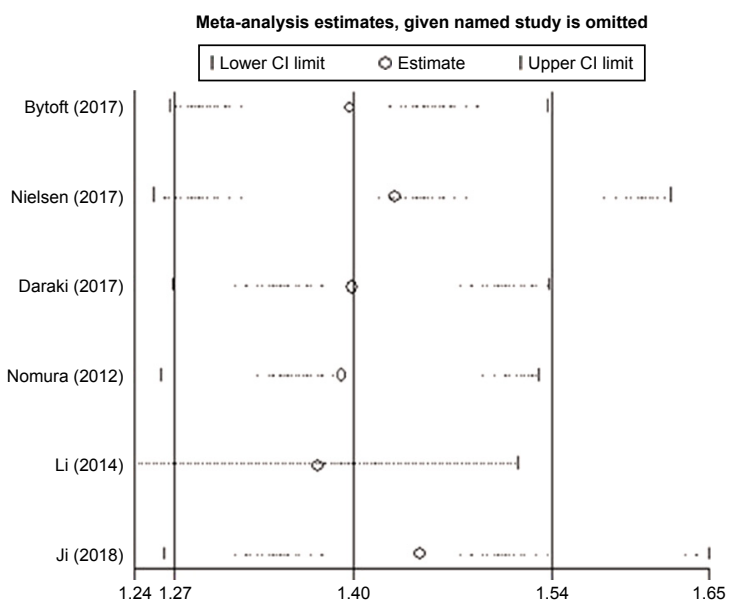

Figure 2 Main results of the meta-analyses.

Notes: (A) Data combination of case-control studies; (B) sensitivity analysis on case-control studies; (C) data combination of cohort studies; and (D) sensitivity analysis on cohort studies. Weights are from random-effects analysis.

Abbreviations: ES, effect size; GDM, gestational diabetes mellitus; TID, type I diabetes; T2D, type 2 diabetes.

with ADHD, particularly in females. ${ }^{14}$ Considering that ADHD has an approximate sex ratio of 3:1 during childhood and displays sex-specific manifestations, ${ }^{23}$ a sex-specific mechanism may underlie the relationship between ADHD and maternal immune disease, such as diabetes. Among the included studies, only one investigation ${ }^{14}$ separately reported the association of maternal diabetes with ADHD in males and females. Although insignificant findings were shown in both males and females, ${ }^{14}$ further studies exploring the difference between gender are encouraged for a better understanding of ADHD etiology.

Social factors have been suggested to play a crucial role on maternal diabetes and glycemic control, thus mediating the onset of ADHD. Most the included studies ${ }^{8,13,14,20,21}$

Table 4 Main results of the meta-analyses

\begin{tabular}{|c|c|c|c|c|c|c|c|c|c|c|}
\hline \multirow[t]{2}{*}{ Analysis } & \multirow{2}{*}{$\begin{array}{l}\text { No of } \\
\text { subjects }\end{array}$} & \multirow{2}{*}{$\begin{array}{l}\text { Statistical } \\
\text { model }\end{array}$} & \multicolumn{3}{|l|}{ Data pooling } & \multicolumn{2}{|c|}{ Heterogeneity } & \multicolumn{2}{|c|}{ Publication bias } & \multirow{2}{*}{$\begin{array}{l}\text { Sensitivity } \\
\text { analysis }\end{array}$} \\
\hline & & & Effect size & $t$-value & $P$-value & $I^{2}(\%)$ & $P$-value & $t$-value & $P$-value & \\
\hline Overall OR & $4,836,263$ & Random & $1.20(0.96-1.49)$ & 1.62 & 0.105 & 76.5 & 0.002 & 1.67 & 0.194 & Stable \\
\hline Overall RR & $2,382,640$ & Fixed & $1.40(1.27-1.54)$ & 6.89 & $<0.001$ & 31.8 & 0.197 & 6.56 & 0.003 & Stable \\
\hline GDM RR & 985,984 & Fixed & $2.00(\mathrm{I} .42-2.8 \mathrm{I})$ & 4.00 & $<0.001$ & 0.0 & 0.446 & 4.99 & 0.038 & Stable \\
\hline GDM in Caucasian RR & 985,014 & Fixed & $2.64(1.26-5.56)$ & 2.56 & 0.011 & 0.0 & 0.369 & 3.30 & 0.187 & Stable \\
\hline
\end{tabular}

Abbreviations: GDM, gestational diabetes mellitus; RR, risk ratio. 

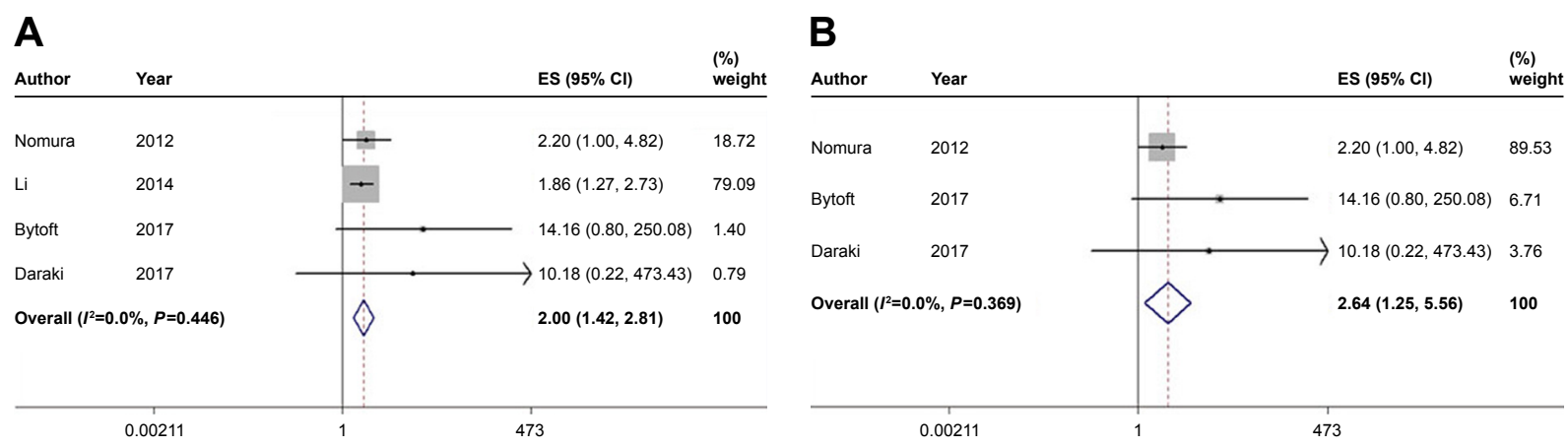

Figure 3 Meta-analyses of the subjects from (A) mothers with GDM or (B) Caucasian mothers with GDM. Abbreviations: ES, effect size; GDM, gestational diabetes mellitus.

considered family socioeconomic status (SES), such as parental income, occupation, education, and single parent status, as a confounding variable and thereby adjusted the result. It is widely accepted that parental socioeconomic disadvantage is a risk factor for ADHD in children, ${ }^{24}$ which is likely mediated by factors linked to low SES such as parental mental disorders. Diabetic mothers, especially for those with complications, are linked to low SES because those women might have limited ability to work and have lower disposable income, resulting in a relatively deprived environment for children. Apart from socioeconomic deprivation, lifestyle, access to health food, regular physical activity, public insurance, etc, are also linked to glycemic control..$^{25}$ Overall, it is recommended to record social factors and consider them as confounders when examining the true effect of maternal diabetes on ADHD in children. So the adjusted results were extracted and combined if available.

A new insight into the impact of in utero hyperglycemia on fetus brain development emphasizes that maternal diabetes may increase the vulnerability to psychiatric disorder later in life by interacting with other environmental insult during pregnancy. Animal model study has demonstrated that GDM and viral infection concurrently produce a novel transcriptional profile, and these novel transcriptional changes are associated with pathways implicated in psychiatric disorders. ${ }^{26}$ This finding indicates that GDM may have the potential to prime the fetus for an exacerbating response to infection, which is a common environmental stress during pregnancy.

The mechanism of ADHD also has some endocrinological components. It has been shown that patients with T1D are more likely to receive ADHD diagnosis. ${ }^{27}$ Interestingly, adolescents and young adults with ADHD are reportedly to be more likely than non-ADHD controls to develop T2D in later life. ${ }^{28}$ There is an ongoing discussion whether thyroid hormone system is involved in the development of ADHD, and there is a link between thyroid hormones and diabetes risk. ${ }^{29}$ In has been observed that thyroid-stimulating hormone levels are slightly increased among ADHD patients. ${ }^{30}$ This finding is further validated by transgenic mouse that expresses a human mutant thyroid receptor $\beta 1$, which manifests typical syndromes of ADHD such as impulsive and inattentive. ${ }^{31}$

The National Institute for Health and Care Excellence accredited guidelines for ADHD management emphasized the importance of a holistic approach to managing ADHD. ${ }^{32}$ A comprehensive shared treatment plan should address psychological, behavioral, and occupational or educational needs. ${ }^{32}$ The guideline recommends an ADHD-focused group parent-training program to parents or carers as first-line treatment for children under 5 years with ADHD. Pharmacological medication is offered to children aged 5 years and over, young people, and adults, only if ADHD symptoms are still causing a significant impairment in interpersonal relationships, education and occupational attainment, or risk awareness. ${ }^{32}$ However, a network meta-analysis of randomized trials indicated that the therapeutic effects of cognitive training, neurofeedback, antidepressants, antipsychotics, dietary therapy, fatty acids, and other complementary and alternative medicine were uncertain due to limited evidences. ${ }^{33}$

The present studies consisted of cohort, cross-sectional, and case-control studies. Heterogeneity existed as a significant limitation for this review because of differences in ADHD definition, ethnicity, type of maternal diabetes, and methodology used. In order to reduce the heterogeneity between studies included in the review, the studies that were similar regarding design and measurement were combined. Another noteworthy fact was that we included the studies in which prescriptions were used as proxy for the diagnosis of ADHD, ${ }^{8,14,19}$ and thus selection bias might occur. Due to various health care systems and policies in different countries, medications are accepted by different subgroups of children who may have 
been diagnosed with ADHD. However, medication for ADHD does not necessarily mean a clinical diagnosis has been given. Therefore, the identification of ADHD cases in further studies should be based on a standardized scale so that the selection bias could be diminished.

\section{Conclusion}

Maternal diabetes, especially for GDM, is probably a risk factor for ADHD in Caucasian race. This result emphasizes the importance of good glycemic control in diabetic mothers throughout pregnancy and not only in the first trimester. The attention on gender difference, the clear record of significant confounders, and the accurate diagnosis of ADHD cases are urgently needed in the further studies.

\section{Disclosure}

The authors report no conflicts of interest in this work.

\section{References}

1. Thomas R, Sanders S, Doust J, Beller E, Glasziou P. Prevalence of attention-deficit/hyperactivity disorder: a systematic review and metaanalysis. Pediatrics. 2015;135(4):e994-e1001.

2. Beau-Lejdstrom R, Douglas I, Evans SJW, Smeeth L. Latest trends in ADHD drug prescribing patterns in children in the UK: prevalence, incidence and persistence. BMJ Open. 2016;6(6):e010508.

3. Grimm O, Kittel-Schneider S, Reif A. Recent developments in the genetics of ADHD. Psychiatry clin Neurosci. 2018;72(9):654-672.

4. Polańska K, Jurewicz J, Hanke W. Exposure to environmental and lifestyle factors and attention-deficit/hyperactivity disorder in children - a review of epidemiological studies. Int J Occup Med Environ Health. 2012;25(4):330-355.

5. Schroeder SR. Mental retardation and developmental disabilities influenced by environmental neurotoxic insults. Environ Health Perspect. 2000;108(Suppl 3):395-399.

6. Bytoft B, Knorr S, Vlachova Z, et al. Long-term cognitive implications of intrauterine hyperglycemia in adolescent offspring of women with type 1 diabetes (the EPICOM study). Diabetes Care. 2016;39(8):1356-1363.

7. Ornoy A, Reece EA, Pavlinkova G, Kappen C, Miller RK. Effect of maternal diabetes on the embryo, fetus, and children: congenital anomalies, genetic and epigenetic changes and developmental outcomes. Birth Defects Res C Embryo Today. 2015;105(1):53-72.

8. Instanes JT, Halmøy A, Engeland A, Haavik J, Furu K, Klungsøyr K. Attention-deficit/hyperactivity disorder in offspring of mothers with inflammatory and immune system diseases. Biol Psychiatry. 2017;81(5): 452-459

9. Banik A, Kandilya D, Ramya S, Stünkel W, Chong Y, Dheen S. Maternal factors that induce epigenetic changes contribute to neurological disorders in offspring. Genes (Basel). 2017;8(6):150.

10. Nomura Y, Marks DJ, Grossman B, et al. Exposure to gestational diabetes mellitus and low socioeconomic status: effects on neurocognitive development and risk of attention-deficit/hyperactivity disorder in offspring. Arch Pediatr Adolesc Med. 2012;166(4):337-343.

11. Li J, Zhong H, Liang ZC, Xy L. Study on correlation between gestational diabetes merged pregnancy-induced hypertension and hyperactivity syndrome in children. J Clin Exp Med. 2014;13(19):1583-1585.

12. Nielsen PR, Benros ME, Dalsgaard S. Associations between autoimmune diseases and attention-deficit/hyperactivity disorder: a nationwide study. J Am Acad Child Adolesc Psychiatry. 2017;56(3):234-240.e1.
13. Ji J, Chen T, Sundquist J, Sundquist K. Type 1 diabetes in parents and risk of attention deficit/hyperactivity disorder in offspring: a populationbased study in Sweden. Diabetes Care. 2018;41(4):770-774.

14. Hegvik TA, Instanes JT, Haavik J, Klungsøyr K, Engeland A. Associations between attention-deficit/hyperactivity disorder and autoimmune diseases are modified by sex: a population-based cross-sectional study. Eur Child Adolesc Psychiatry. 2018;27(5):663-675.

15. Moher D, Liberati A, Tetzlaff J, Altman DG; PRISMA Group. Preferred reporting items for systematic reviews and meta-analyses: the PRISMA statement. PLoS Med. 2009;6(7):e1000097.

16. Egger M, Smith GD, Schneider M, Minder C. Bias in meta-analysis detected by a simple, graphical test. BMJ. 1997;315(7109):629-634.

17. Higgins JPT, Thompson SG. Quantifying heterogeneity in a metaanalysis. Stat Med. 2002;21(11):1539-1558.

18. Dersimonian R, Laird N. Meta-analysis in clinical trials. Control Clin Trials. 1986;7(3):177-188.

19. Bytoft B, Knorr S, Vlachova Z, et al. Assessment of attention deficits in adolescent offspring exposed to maternal type 1 diabetes. PLoS One. 2017;12(1):e0169308

20. Daraki V, Roumeliotaki T, Koutra K, et al. Effect of parental obesity and gestational diabetes on child neuropsychological and behavioral development at 4 years of age: the Rhea mother-child cohort, Crete, Greece. Eur Child Adolesc Psychiatry. 2017;26(6):703-714.

21. Schmitt J, Romanos M. Prenatal and perinatal risk factors for attentiondeficit/hyperactivity disorder. Arch Pediatr Adolesc Med. 2012;166(11): 1074-1075.

22. Hedderson M, Ehrlich S, Sridhar S, Darbinian J, Moore S, Ferrara A. Racial/ethnic disparities in the prevalence of gestational diabetes mellitus by BMI. Diabetes Care. 2012;35(7):1492-1498.

23. Faraone SV, Asherson P, Banaschewski T, et al. Attention-deficit/ hyperactivity disorder. Nat Rev Dis Primers. 2015;49:15020.

24. Russell AE, Ford T, Williams R, Russell G. The association between socioeconomic disadvantage and attention deficit/hyperactivity disorder (ADHD): a systematic review. Child Psychiatry Hum Dev. 2016;47(3): $440-458$.

25. Colicchia LC, Parviainen K, Chang JC. Social contributors to glycemic control in gestational diabetes mellitus. Obstet Gynecol. 2016;128(6): 1333-1339.

26. Money KM, Barke TL, Serezani A, et al. Gestational diabetes exacerbates maternal immune activation effects in the developing brain. $\mathrm{Mol}$ Psychiatry. 2018;23(9):1-9.

27. Kapellen TM, Reimann R, Kiess W, Kostev K. Prevalence of medically treated children with ADHD and type 1 diabetes in Germany Analysis of two representative databases. J Pediatr Endocrinol Metab. 2016;29(11):1293-1297.

28. Chen MH, Pan TL, Hsu JW, et al. Risk of type 2 diabetes in adolescents and young adults with attention-deficit/hyperactivity disorder. J Clin Psychiatry. 2018;79(3). pii: 17m11607.

29. Crunkhorn S, Patti ME. Links between thyroid hormone action, oxidative metabolism, and diabetes risk? Thyroid. 2008;18(2):227-237.

30. Cakaloz B, Akay AP, Bober E, Yulug B. Thyroid function and oppositional defiant disorder: more than a coincidence in prepubertal boys with attention-deficit hyperactivity disorder? J Neuropsychiatry Clin Neurosci. 2011;23(2):E9-E10.

31. Siesser WB, Zhao J, Miller LR, Cheng SY, McDonald MP. Transgenic mice expressing a human mutant betal thyroid receptor are hyperactive, impulsive, and inattentive. Genes Brain Behav. 2006;5(3):282-297.

32. NICE. Attention deficit hyperactivity disorder: diagnosis and management 2018. Available from: www.nice.org.uk/guidance/NG87. Accessed January 1, 2019.

33. Catalá-López F, Hutton B, Núñez-Beltrán A, et al. The pharmacological and non-pharmacological treatment of attention deficit hyperactivity disorder in children and adolescents: a systematic review with network meta-analyses of randomised trials. PLoS One. 2017; 12(7):e0180355. 


\section{Publish your work in this journal}

Neuropsychiatric Disease and Treatment is an international, peerreviewed journal of clinical therapeutics and pharmacology focusing on concise rapid reporting of clinical or pre-clinical studies on a range of neuropsychiatric and neurological disorders. This journal is indexed on PubMed Central, the 'PsycINFO' database and CAS, and is the official journal of The International Neuropsychiatric Association (INA). The manuscript management system is completely online and includes a very quick and fair peer-review system, which is all easy to use. Visit http://www.dovepress.com/testimonials.php to read real quotes from published authors.

\footnotetext{
Submit your manuscript here: http://www.dovepress.com/neuropsychiatric-disease-and-treatment-journal
} 